\title{
Cardiovascular health knowledge and preventive practices in people living with HIV in Kenya
}

Tecla M. Temu ${ }^{1,2^{*}}$, Nicholas Kirui ${ }^{1,4}$, Celestine Wanjalla ${ }^{5}$, Alfred M. Ndungu' ${ }^{6}$, Jemima H. Kamano ${ }^{1,3,4}$, Thomas S. Inui ${ }^{1,3,4,7}$ and Gerald S. Bloomfield ${ }^{8}$

\begin{abstract}
Background: Traditional cardiovascular disease (CVD) risk factors contribute to increase risk of CVD in people living with HIV (PLWH). Of all world regions, sub-Saharan Africa has the highest prevalence of HIV yet little is known about PLWH's CVD knowledge and self- perceived risk for coronary heart disease (CHD). In this study, we assessed PLWH's knowledge, perception and attitude towards cardiovascular diseases and their prevention.

Methods: We conducted a cross-sectional study in the largest HIV care program in western Kenya. Trained research assistants used validated questionnaires to assess CVD risk patterns. We used logistic regression analysis to identify associations between knowledge with demographic variables, HIV disease characteristics, and individuals CVD risk patterns.

Results: There were 300 participants in the study; median age (IQR) was 40 (33-46) years and $64 \%$ women. The prevalence of dyslipidemia, overweight and obesity were $70 \%, 33 \%$ and $8 \%$, respectively. Participant's knowledge of risk factors was low with a mean (SD) score of 1.3 (1.3) out of possible 10. Most (77.7 \%) could not identify any warning signs for heart attack. Higher education was a strong predictor of CVD risk knowledge $(6.72,95 \% \mathrm{Cl}$ 1.98-22.84, $P<0.0001)$. Self-risk perception towards CHD was low (31 \%) and majority had inappropriate attitude towards CVD risk reduction.

Conclusion: Despite a high burden of cardiovascular risk factors, PLWH in Kenya lack CVD knowledge and do not perceived themselves at risk for CHD. These results emphasis the need for behavior changes interventions to address the stigma and promote positive health behaviors among the high risk HIV population in Kenya.
\end{abstract}

Keywords: HIV, Cardiovascular diseases, Sub Saharan Africa, Kenya, Knowledge, Perception

\section{Background}

Over 22.5 million people living with human immunodeficiency virus (PLWH) reside in sub-Sahara Africa (SSA), representing $68 \%$ of the global HIV burden [1]. At the same time, CVD accounts for $11 \%$ of deaths in SSA and the number of deaths due to CVD have increased by $81 \%$ between 1990 and 2013 [2, 3]. The burden of CVD is expected to double by 2020 [3]. Half of CVD deaths in SSA occur among people aged between 30 and 69, which is

\footnotetext{
* Correspondence: Tecla.m.temu@vanderbilt.edu

'Department of Medicine, School of Medicine, College of Health Sciences,

Moi University, Eldoret, Kenya

${ }^{2}$ Department of Epidemiology, Brown University School of Public Health,

Providence, RI, USA

Full list of author information is available at the end of the article
}

10 years earlier than in North America and Europe [http://www.ichealth.org]. Mortality and morbidity in this age group has major social and economic consequences depriving families of parents, work places of employees and communities of leaders.

There is a greater than expected risk of CVD in PLWH $[4,5]$. The commonly reported CVD manifestations include carotid atherosclerosis, large vessel vascular disease, and coronary artery disease [6]. Increased CVD risk in PLWH is attributed to chronic inflammation, immune activation associated with HIV-infection, opportunistic infections and traditional CVD risk factors including dyslipidemia associated with antiretroviral therapy (ART) [6]. Although the overall risks and benefit of ART for 
reducing cardiovascular events is still controversial, continuous ART has been associated with better CVD outcomes than the ART interruption as demonstrated by the Strategies for Management of Antiretroviral Therapy (SMART) study [7]. Therefore, among the many factors, modifiable risk factors such hypertension, obesity, dyslipidemia, smoking and lack of physical activity seem to play a central role in accelerating the risk of CVD in this population [8]. Acute myocardial infarction risk increases exponentially with each additional cardiovascular risk factor in PLWH compared to having no CVD risk factors [8]. Most studies reporting high CVD risk in PLWH have been performed in developed countries $[9,10]$. The few data from SSA also suggest greater CVD risk in PLWH [11-14].

Knowledge of CVD risk factors is a critical prerequisite for an individual to make behavioral changes leading towards optimal cardiovascular health $[15,16]$. Patients with good knowledge of heart attack warning signs are more likely to present for treatment earlier with better outcomes [17]. Individuals with a greater knowledge of coronary heart disease (CHD) risk factors also have higher perceived risk [18]. According to health behavior model, individuals with high perceived risk for CVD are more likely to adopt healthy behaviors such as weight loss and smoking cessation [16]. Cultural biases and socio- economics are of great significance in resource-limited areas such as SSA and these factors are lacking in many studies in this field [19]. Therefore, measuring knowledge level of PLWH on CVD is a crucial step in the designing of future prevention strategies for PLWH in SSA. In this study, we examined PLWH's CVD risk factor profile, knowledge of CVD risk factors and warning sign of heart attack, risk perception and currents practices towards cardiovascular health in Kenya, a country with an overall $6 \%$ prevalence of HIV and rising CVD burden [20]. We are not aware of any studies that have examined the knowledge of CVD in PLWH in SSA. Results from this study will be used to guide public health interventions to reduce cardiovascular disease risk in PLWH in SSA.

\section{Methods}

\section{Ethical consideration}

The Institutional Research and Ethics Committee of Moi University School of Medicine approved the study. All participants provided a written informed consent. Any findings that were felt to warrant immediate medical attention were reported to the participant and their physician.

\section{Study site}

Participants were recruited from the outpatient HIV clinic of Moi Teaching and Referral Hospital (MTRH) in Eldoret within the Academic Model Providing Access to Healthcare (AMPATH) program which provides care to $>150,000$ adults and children living with HIV/AIDS throughout
Western Kenya [21]. The AMPATH program is a collaboration between MTRH, Moi University School of Medicine, and a consortium of North American universities that focuses on improving the health of the people of Western Kenya as previously described [22]. This site was chosen because of its broad mix of patients including a mix of urban middle class, urban poor and the rural population. Moi University is home to an NHLBI-sponsored Cardiovascular and Pulmonary Disease Center of Excellence in Cardiovascular and Pulmonary Diseases (COE) [23], and is the hub of clinical research in cardiopulmonary diseases in Western Kenya [24]. HIV-infected patients fulfilling Kenyan national criteria for ART are started on treatment and are seen monthly at the AMPATH clinic. Criteria for starting ART at the time of the study included all adult PLWH with CD4 $\mathrm{T}$ cell count $<350$ cells $/ \mathrm{mm}^{3}$ irrespective of WHO stage and stage III/ IV disease regardless of CD4 T cell count. The first-line ART regimen consisted of either tenofovir/ lamivudine or zidovudine/lamivudine + nevirapine or efavirenz. Protease inhibitors (PIs) were only given as second-line ART in accordance with Kenya national guidelines for antiretroviral drug therapy [25].

\section{Study population and design}

As part of a larger study to assess the prevalence of cardiovascular risk factors in PLWH, we conducted a descriptive cross-sectional study to investigate knowledge, attitude and self-risk perception towards CVD in a sample of PLWH in Western Kenya attending AMPATH HIV clinic. The study sample was a convenience sample of patients consisting of two groups of adults (aged $\geq 18$ years old) with a diagnosis of HIV; adult PLWH not yet on ART, and adult PLWH on ART regardless of the length of time they have been on ART. Participants were required to be able give a written informed consent and respond to questions. Exclusion criteria included any patients with recorded history of CVD. Consecutive adult PLWH who met the criteria were asked to participate.

\section{Data collection}

Between July to September 2014, research assistants invited patients who presented for their routine clinic appointment to participate in the study. Data were collected by structured questionnaires, physical examination and venous blood sample analysis. A trained research assistant administered the questionnaire in English, Swahili or a local language (Additional file 1). Each interview lasted approximately $30 \mathrm{~min}$ followed by physical measurements. Participants were asked return to clinic the following day after fasting for eight hours for biochemical assessments. The research participants were expected to complete all the components of the research examination on the second visit whenever possible. Participants received compensation to cover for transportation. 


\section{Questionnaire}

Items in the questionnaire were constructed from multiple validated surveys [26-28]. The draft questionnaire was piloted with 25 people to measure its validity prior to administering to the study population. The final questionnaire contained three sections: social and demographic characteristics, knowledge of CVD and CHD; and attitude and perception towards CHD and CHD prevention (Additional file 1). Open ended and prompt questions were incorporated in all three sections.

\section{Social demographic characteristics and medical history}

Included information on age, sex, marital status, education level, medical history including HIV duration and family history of CVD. Cardiovascular risk factors assessment was done using items from the World Health Organization non -communicable disease STEPwise approach to CVD risk factor surveillance [29]. Information on use of ART, the type of ART regime used and latest CD4+ lymphocyte count was obtained from the participant's medical records.

\section{Knowledge of CVD and CHD}

Participants' were asked to list up to ten CVD risks factors and seven CHD warning signs. Responses were graded as correct or incorrect using guidelines from the American Heart Association as the gold standard [30]. Acceptable responses included age, high blood pressure (BP), high cholesterol, smoking, obesity, family history, diabetes, stress, alcohol use, and physical inactivity. Acceptable warning symptoms of heart attack included dizziness, shortness of breath, sweating, loss of consciousness, chest pain, nausea/ vomiting, and pain in the teeth, arm, or jaw. Each response was scored 0 for a wrong answer or 1 for the correct answer, with a minimum score of 0 and a maximum score of 10 points or 7 points for CVD risk factors and CHD warning signs, respectively. Participants were also asked to identify action they would take in case they suspected a heart attack.

\section{Attitude and Perception towards CVD and CHD prevention}

This section contained questions to assess participants' self-perceived risk for heart diseases, perceived seriousness of CHD, attitude and beliefs, and current practices for CHD prevention. In addition, participant's ways of accessing health information was also recorded.

\section{Physical measurements}

Height, weight, waist, and hip circumference were measured. Body mass index (BMI) was calculated using the measured weight and height $\left(\mathrm{kg} / \mathrm{m}^{2}\right)$. Blood pressure was measured using an automated digital sphygmomanometer (Omron Hem 712c, Omron Healthcare, Kyoto,
Japan). Bilateral BP was taken twice, with two-minute interval between measurements. High BP was defined as systolic $\mathrm{BP} \geq 140 \mathrm{~mm} \mathrm{Hg}$, diastolic $\mathrm{BP} \geq 90 \mathrm{~mm} \mathrm{Hg}$, or currently on antihypertensive drug treatment. Central obesity was defined as waist circumference of $\geq 80 \mathrm{~cm}$ (women) and $\geq 90 \mathrm{~cm}$ (men).

\section{Biochemical assessments}

Blood samples from individuals fasting for $>8 \mathrm{~h}$ were collected by venipuncture in BD vacutainer tubes. Samples were typically processed within $4 \mathrm{~h}$ of collection. A five-milliliter $\mathrm{BD}$ vacutainer tube was centrifuged at $3000 \mathrm{rpm}$ for $4 \mathrm{~min}$ and serum was collected. Biochemical analysis was performed using the COBAS Integra 400 plus chemistry analyzer to determine blood glucose (FBG) and lipid profiles from the serum sample. Diabetes mellitus was defined as FBG $\geq 126 \mathrm{mg} / \mathrm{dl}$ and dyslipidemia was defined as total cholesterol (TC) $\geq 200 \mathrm{mg} /$ $\mathrm{dl}$ or HDL-C $<40 \mathrm{mg} / \mathrm{dl}$, LDL-C $\geq 130 \mathrm{mg} / \mathrm{dl}$, and triglycerides $>350 \mathrm{mg} / \mathrm{dl}$ by the American Heart Association and American College of Cardiology Foundation [31].

\section{Statistical analysis}

The analysis was completed using $\mathrm{SAS}^{\oplus}$ University Edition. Descriptive data are presented as means and percentage. Continuous data are presented as median and interquartile range (IQR). Chi Square tests were performed to investigate the association of knowledge level with categorical variables. Logistic regression analyses were done to identify univariate associations between the ability to identify risk factors for heart attack and warning symptoms. A multivariate logistic regression model was fit to analyze these associations after controlling for demographic variables, HIV disease characteristics, and individuals CVD risk patterns. Chi-square test was performed to measure association between heart disease risk perception and CVD risk status. CVD risk status was defined as a binary outcome as having one or more of the established risk factors for CVD. Statistical significance was set at $P<0.05$.

\section{Results}

\section{Demographic and health characteristics}

Of 300 participants, 192 (64\%) were female and the median (IQR) age was 39. 7(33.6 - 46.3) years. Over half (55\%) were on ART, $38.6 \%$ had more that 8 years of formal education. The median (IQR) HIV and ART use duration were $4.6(1.7-7.9)$ and $4.8(2.7-7.8)$ years, respectively. Additional demographic characteristics are reported in Table 1.

Figure 1 shows the distribution of CVD risk factors among participants. The prevalence of dyslipidemia, central obesity, and overweight/obese was $70 \%, 57 \%$, and $41 \%$ respectively. Average (SD) BMI was 24.4 (5.6). Mean 
Table 1 Characteristics of the study participants

\begin{tabular}{|c|c|}
\hline Characteristics & Total $(n=300)$ \\
\hline Median age, y (IQR) & $39.7(33.6-46.3)$ \\
\hline Female, n (\%) & $192(64)$ \\
\hline \multicolumn{2}{|l|}{ Overall age data (y) } \\
\hline $18-39$ & $146(49)$ \\
\hline $40-59$ & $142(47)$ \\
\hline $60-80$ & $12(4)$ \\
\hline \multicolumn{2}{|l|}{ Education, n (\%) } \\
\hline Tertiary (>12) & $40(13.3)$ \\
\hline Secondary ( 9 - 12 grade) & $76(25.3)$ \\
\hline Primary (0-8 grade) & $131(43.7)$ \\
\hline Never attended school & $53(17.7)$ \\
\hline \multicolumn{2}{|l|}{ Marital status, n (\%) } \\
\hline Single & $45(15)$ \\
\hline Married & $235(78.3)$ \\
\hline Widowed & $16(5.4)$ \\
\hline Separated & $4(1.3)$ \\
\hline \multicolumn{2}{|l|}{ Current professional status, n (\%) } \\
\hline Employed & $74(25)$ \\
\hline Self-employed & $132(44)$ \\
\hline Unemployed & $94(31)$ \\
\hline \multicolumn{2}{|l|}{ Family History, n (\%) } \\
\hline High Cholesterol & $2(0.7)$ \\
\hline Diabetes & $9(3)$ \\
\hline High blood pressure & $4(1.3)$ \\
\hline Myocardial infraction & $1(0.3)$ \\
\hline \multicolumn{2}{|l|}{ HIV related characteristics } \\
\hline \multicolumn{2}{|l|}{ WHO HIV stage, n (\%) } \\
\hline Stage $1 \& 2$ & $204(68)$ \\
\hline Stage $3 \& 4$ & $96(32)$ \\
\hline Median HIV infection duration (y), IQR & $4.6(1.7-7.9)$ \\
\hline Median ART duration (y), IQR & $4.8(2.7-7.8)$ \\
\hline Current CD $+\mathrm{T}$ cell, (cells/mm³), SD & $413(215)$ \\
\hline Protease inhibitor use & $11(3.7)$ \\
\hline ART-naive & $136(45)$ \\
\hline
\end{tabular}

ART antiretroviral therapy

(SD) total cholesterol, LDL cholesterol and HDL was $174 \mathrm{mg} / \mathrm{dl}(3.8), 104 \mathrm{mg} / \mathrm{dl} \mathrm{[18]} \mathrm{and} 50 \mathrm{mg} / \mathrm{dl}$ [22] respectively. Alcohol and tobacco use were reported in $20 \%$ and $3.3 \%$ of the participants, respectively. Most participants (92\%) reported being physically active and less than $3 \%$ were found to have high BP or diabetes. Mean (SD) fasting glucose for our participants was $90 \mathrm{mg} / \mathrm{dl}$ [17]. None of our participants reported receiving treatment for dyslipidemia, hypertension or diabetes. Almost $44 \%$ of the participants had two or more risk factors. Women were likely to

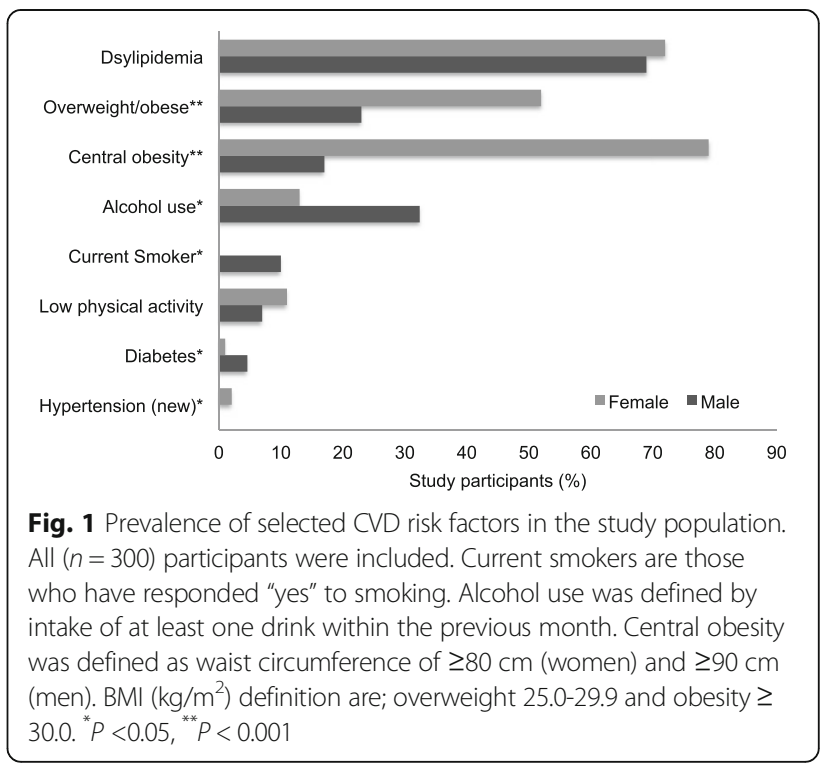

have overweight/obesity $(P=0.03)$, to have raised BP $(P<$ $0.0001)$, central obesity $(P=0.001)$, and less likely to report tobacco use $(P<0.0001)$, alcohol use $(P=0.001)$ than men.

\section{Knowledge of CVD risk factors}

In an open-ended question, participants were asked to list risk factors for CVD. Table 2 shows unprompted responses for the perceived risk factors of CVD. Knowledge of CVD risk factor was assessed as a continuous variable. The mean (SD) score for CVD risk factors identified by participants was 1.3 (1.3) out of possible 10. The most common risk factor identified by the group was stress (74 \%) followed by physical inactivity (13\%) and obesity/ overweight (9\%). Less than $10 \%$ of participants correctly identified an established biological risk factor for CVD while sixteen percent of participants could not identify any risk factor at all (Fig. 2).

Table $\mathbf{2}$ CVD risk factors as identified by participants

\begin{tabular}{ll}
\hline Risk factors & $\mathrm{N}(\%)$ \\
\hline Don't know & $47(16)$ \\
Don't know & $47(16)$ \\
Stress & $221(73.7)$ \\
Physical inactivity & $38(12.6)$ \\
Overweight/obese & $28(9.3)$ \\
Raised blood pressure & $27(9.0)$ \\
Alcohol overuse & $23(7.6)$ \\
Diabetes & $15(5.0)$ \\
High Cholesterol & $15(5.0)$ \\
Smoking/Tobacco use & $12(4.0)$ \\
Age & $7(2.3)$ \\
Family history & $4(1.3)$ \\
\hline
\end{tabular}




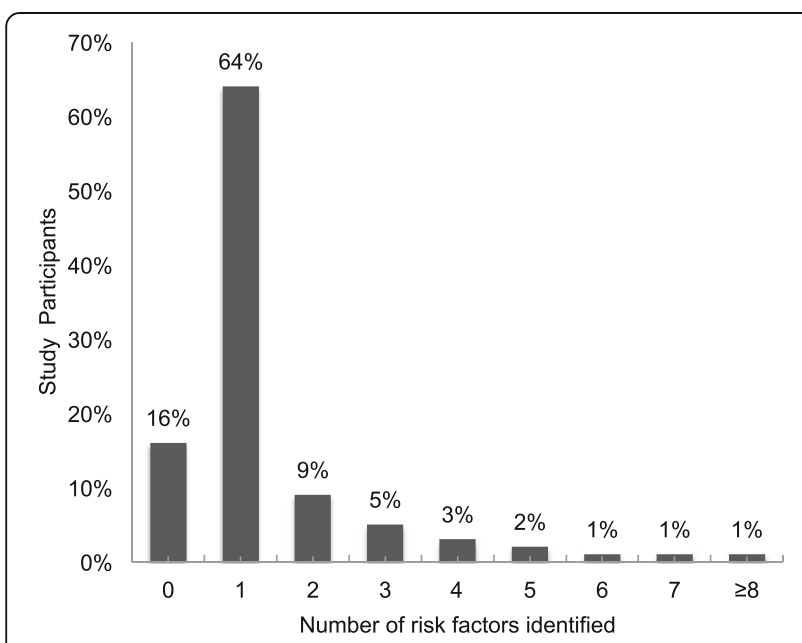

Fig. 2 CVD risk factors as identified by participants

In univariate analysis, factors significantly associated with ability to identify $\geq 1$ CVD and CVD risk factor were $>12$ years of education (OR 4.18, $95 \%$ CI 1.7410.07, $P<0.0001$ ) and family history of diabetes (OR 5.21, $95 \%$ CI $0.99-27.37, P=0.05)$. However in the multivariable analysis, only $>12$ years of education was independently associated with the ability to identify $\geq 1$ CVD risk factor (OR 6.72, $95 \%$ CI 1.98-22.84, $P<$ $0.000)$. No significant association was found between risk knowledge and risk status $(P=0.26)$.

\section{Knowledge of heart attack symptoms}

Participants were also asked to list heart attack warning signs. Table 3 shows participant knowledge of heart attack symptoms. The mean (SD) score for heart attack symptoms identified by the group was $0.28(0.6)$ out of 7 . With the exception of loss of consciousness and difficulty breathing identified by $10 \%$ of participants, a very small proportion $(<3 \%)$ could identify the other established warning signs. Over three quarters of participants (77 \%) could not identify one warning sign (Fig. 3). No significant associations were observed between heart attack symptoms knowledge (defined as identification of $\geq$

Table 3 Heart attack warning signs as identified by participants

\begin{tabular}{ll}
\hline Warning signs & $\mathrm{N}(\%)$ \\
\hline Don't know & $233(77.3)$ \\
Loss of consciousness & $31(10.3)$ \\
Dyspnea & $30(10.0)$ \\
Dizziness or light headedness & $10(3.3)$ \\
Chest pain & $5(1.7)$ \\
Excessive sweating & $3(1.0)$ \\
Nausea/Nomiting & $2(0.7)$ \\
Pain in the teeth, jaw or arm & $2(0.7)$ \\
\hline
\end{tabular}

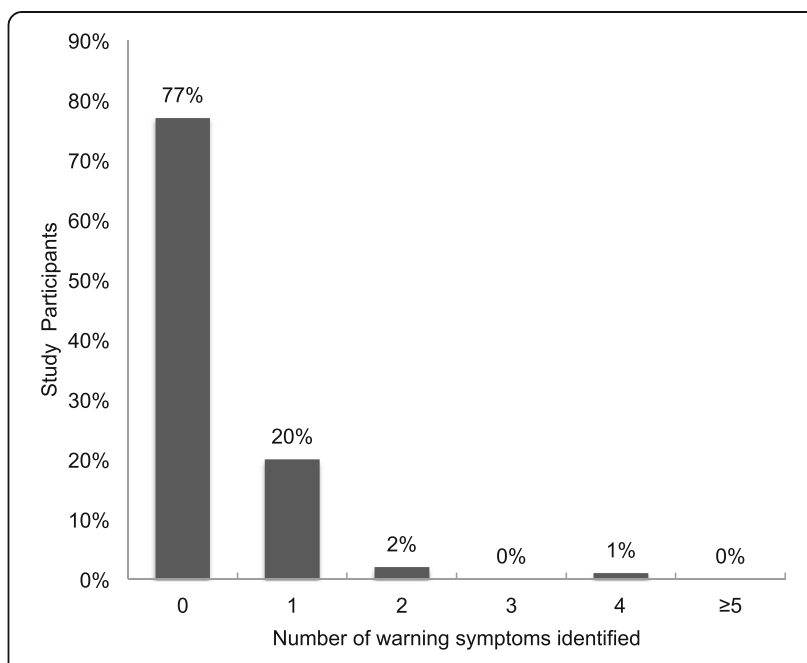

Fig. 3 Heart attack warning signs as identified by participants

1 heart attack warning signs) and the demographic variables. An overwhelming majority reported that if they thought they were having a heart attack they will go to a health care facility (95\%) while the remaining five percent noted they will go to the pharmacy or use traditional medicines.

\section{Perceived seriousness of heart diseases}

Participants were asked in an open-ended question to list the greatest health problem in PLWH. Most (53\%) noted stress, AIDS (17 \%) and depression (14 \%). Heart diseases were noted by $1 \%$ of the participants, all male $(P=0.03)$. When asked to list the leading cause of death in PLWH, opportunistic infections such as tuberculosis were noted by $94 \%$ of participants.

\section{Self-Perceived risk for heart disease}

Only one third of the participants agreed with the statement "I am at high risk for heart disease". Older women were more likely to agree with this statement $(P=0.04)$ than men. Less than half of the participants agreed that CHD is preventable and that changing lifestyle behavior would reduce their chances of developing CHD. No association was found between risk status and self perceived risk to $\mathrm{CHD}(P=0.1937)$. Other beliefs, or and attitude about heart diseases risk and prevention are shown on Table 4.

Preventive practices against CHD were also assessed (Table 4). Less than $4 \%$ had ever had their blood sugar or cholesterol monitored. In addition, $<5 \%$ of participants reported recreational exercise, cutting down on unhealthy foods, or smoking cessation.

\section{Source of heart disease information}

Over half of our participants reported hearing about CHD in the preceding year. Main source of CVD information 
Table 4 Attitude/beliefs towards CHD risk and current practices towards CHD prevention

\begin{tabular}{lll}
\hline Attitudes towards CHD prevention & Number & Percent \\
Changing lifestyle behavior will cut down my & 131 & (41) \\
chances of developing heart diseases & & \\
(HBM Self efficacy) & & \\
I am at risk for heart disease. (HBM perceived risk) & 208 & (31) \\
Heart disease is preventable & 37 & (12) \\
My present weight is too high for my health & 29 & (10) \\
I need to cut down on the amount of food I eat & 27 & (9) \\
Current practices towards cardiovascular health & & \\
In the past year, have you & & \\
Had your blood pressure measured & 300 & (100) \\
Tried to cut down weight & 11 & (4) \\
Increase physical activity/exercise & 9 & (3) \\
Had cholesterol or blood sugar checked & 8 & (3) \\
Attempted to cut down on unhealthy foods & 7 & (2) \\
Reduce/quit smoking & 1 & (1) \\
\hline
\end{tabular}

Questions on attitude towards CHD prevention were based on a 5-scale likert questions (strongly disagree, disagree, I do not know, agree and strongly agree. We classified five options into three categories as 'agree, 'disagree' and 'I do not know'. Only 'strongly agree' and 'agree' answers were merged and presented in the table

were television (51\%), radio (44\%), magazine (19\%) or internet $(4 \%)$ rather than a health care professional $(4 \%)$. Only $3 \%$ reported to have discussed CVD with a health care provider.

\section{Discussion}

Despite the high frequency of CVD risk factors among participants, we found that PLWH in western Kenya had poor knowledge of both CVD risk factors and heart attack warning signs and had low self-perceived risk of CHD. Preventive practices towards health heart were also lacking. Few participants cited heath care professionals as source of CHD information. Our study suggests that CVD knowledge, a critical ingredient in understanding risk, is low. The information obtained from this study can inform future strategies and interventions for CVD prevention in PLWH.

Despite participants being relatively young, the prevalence of multiple CVD risk factors was high. Obesity and dyslipidemia was diagnosed in over half of our patients. One third of our participants had more than one CVD risk factor. Protease inhibitors (PI) have been associated with increased risk of myocardial infarction [32]. It is unlikely that protease inhibitors were the major drivers of obesity and dyslipidemia because less than $4 \%$ of the participants had been on a PI-based regimen. Perhaps other factors, including but not limited to traditional risk factors, chronic inflammation associated with HIV and non-PI based ART regiments may also be responsible. The link between traditional risk factors and atherosclerosis has been well documented in the developed countries [10]. But a recent study in South Africa by Schoffelen et al. demonstrated, for the first time, that subclinical atherosclerosis was strongly associated with traditional risk factors rather than the HIV-related factors in PLWH supporting earlier findings from the developed countries $[8,10,14]$. This report together with our data emphasize the need to integrate CVD risk assessment and implementing cost effective strategies to manage CVD risk factors into the current HIV care in resourcelimited countries.

Knowledge of the established CVD risk factors was very low. This is in accordance with a study conducted in PLWH in the US [33]. The most common risk factor identified in the present study was stress, consistent with findings the general population from non-Western countries [34, 35]. Established risk factors, including those highly prevalent in our HIV population such as high cholesterol levels were only identified by few participants. These results contrast a study assessing knowledge of CVD in PLWH in the USA whereby $>90 \%$ of participants were able to recognize all the established risk factors [36]. In addition, knowledge did not differ by risk status. A potential explanation for our observation is that physicians may not be addressing CVD factors with their patients as our data support. Physicians may regard CVD risk to be very low in PLWH or they may lack awareness of growing threat of CVD in this population. Additionally, the general lack of knowledge may also be the result of underrepresentation of CVD in the health campaigns and biomedical research in SSA [37]. Only $>12$ years of formal education was independently associated with better knowledge of risk factors, consistent with other survey data [34, 35, 38]. People who have attained higher level of education usually have more health literacy than their counterparts. These results highlight the urgent need of tailored CVD educational programs specifically targeting high-risk PLWH with low education and low literacy skills.

Heart attack symptoms knowledge was much lower than risk factor knowledge. No previous studies have assessed heart attack symptoms knowledge in PLWH. Most participants could not recognize any heart attack symptoms; a finding consistent with previously reported data from other developing countries [39]. In this study, a limited number of the participants identify chest pain as a symptom. This finding contrasts other studies that assessed knowledge in highrisk populations whereby chest pain was the most common identified symptom [34]. Although most of the participants indicated that they would visit a health care facility in the event of a heart attack, not recognizing very basic symptoms such as chest pain may cause major delays in seeking care and worse 
clinical outcomes [17]. CVD risk status was not related to heart attack symptoms knowledge.

Over two thirds of our participants were found to have at least one CVD yet perception of personal risk for CHD was remarkably low. Our findings are consistent with similar studies in PLWH in the developed countries [33, 36]. In addition, no association was found between CVD risk status and participants' self perceived risk for the disease. As the health belief model suggested, an individual is likely to take a recommended health action when perceived risk is high. Low perception of CHD risks may inhibit appropriate actions to prevent CHD [16]. It is possible that the general lack of CVD knowledge among participants diminished risk perception [18]. In addition, CVD screening is not a routine part of HIV care in many HIV treatment programs in sub-Saharan Africa therefore prior to this study majority of the participants were not aware of their risk status. As noted in the health belief model, people have a high probability of perceiving themselves at higher risk of disease when the existence of a risk factor is known. Central obesity is also considered a sign of wealth and health by majority of people in Kenya. Therefore, what is considered healthy may not be perceived as such for this population. Another possible explanation for these findings could be the presence of an optimistic, age-related bias. Our study population was made up of relatively young adults and only $16 \%$ were older than 50 years thus they may believe that as individuals they were not at risk for CVD events, which tend to occur most often in an older population. Future studies need to investigate effective ways to communicate CVD risk to PLWH so that these individuals understand their risk of CVD and adopt risk reduction behaviors.

Consistent with overall poor knowledge and low selfperceived risk for heart disease, preventive practices were significantly lacking. Only one out of eleven who indicated that they smoke had attempted to quit smoking. Although over half of our participants were found to be overweight or obese, less than five percent reported actively exercising, attempting to lose weight or modifying their diet to improve their health. Reasons given by participants who reported positive health behaviors were: wanted to feel better and advise form a healthcare professional corresponding to the perceived benefits and cues of action domains of the HBM, respectively.

Knowledge alone is not sufficient to improve cardiovascular health but it is a vital pre-requisite for improving attitude, behaviors and practices towards better cardiovascular health [16]. This is mostly because behavior change is influenced by one's perception and knowledge that those behaviors could cause harm [18]. Since majority of the participants reported using media as a source of medical information, these could be powerful tool for dissemination information on cardiovascular diseases.
There are several limitations to this study. First, this study enrolled a convenience sample consisting of patients who were actively engaged in HIV care. Findings may differ among those not seeking care. Secondly, men were underrepresented in the study. This is partly related to the fact that in this region women tend to seek care more often than men and are readily available to participate in studies. However, given the dearth of women in the largest studies examining the relationship between HIV and CVD, our findings are relevant to an under-represented population of PLWH [8]. Lastly, the use of an open-ended questionnaire could introduce recall bias resulting in underestimation of knowledge stage of the participants.

\section{Conclusion}

This is the first study to assess risk status, knowledge, perception, and practices of PLWH towards CVD prevention in SSA. The study highlights the high prevalence of CVD risk, lack of knowledge of CVD, and inappropriate attitude towards CHD prevention. Our findings have major implications for HIV healthcare providers in Kenya who will bear the brunt of the projected increased in CVD in PLWH in SSA. Alongside CVD treatment modalities, targeted interventions to improve knowledge and perceptions towards CVD are urgently needed in this population. There is great need to integrate CVD risk assessment into the current HIV infrastructure in SSA.

\section{Additional file}

Additional file 1: Questionnaire. (PDF $75 \mathrm{~kb}$ )

\section{Abbreviations}

CVD: Cardiovascular diseases; CHD: Coronary heart diseases; SSA: Sub Saharan Africa; PLWH: People living with HIV; HIV: Human immunodeficiency virus; ART: Antiretroviral therapy; HDL-C: High-density lipoprotein; LDL-C: Low -density lipoprotein.

\section{Competing interest}

The authors declare that they have no competing interests.

\section{Authors' contribution}

TMT developed the original research idea with contributions by NK, GSB, JHK and TSI. TMT and CW did the background literature review. TMT and AMN assembled and managed the databases and performed statistical analysis. TMT and CW drafted the report. TMT, GSB, CW, TSI, AMN, JHK critically reviewed the report. All authors have read and approved the final version.

\section{Acknowledgement}

This project was supported by NIH Research Training Grant \# R25 TW009337 funded by the Fogarty International Center. We acknowledge Edmond Richmond, Belinda Korir and the Cardiovascular and Pulmonary Disease Center of Excellence in Kenya (HHSN268200900031C), President's Emergency Plan for AIDS Relief (PEPFAR)/USAID (AID-623-A-12-0001). TT is supported by grant 5R24TW009337 from the Fogarty. International Center of the National Institutes of Health $(\mathrm{NIH})$ and the Bureau of Educational and Cultural Affairs of the US Department of State. GB is supported by grant K01TW008407 from the Fogarty International Center of the National Institutes of Health $(\mathrm{NIH})$. 
The content is solely the responsibility of the authors and does not necessarily represent the official views of the National Institutes of Health.

\section{Author details}

'Department of Medicine, School of Medicine, College of Health Sciences, Moi University, Eldoret, Kenya. ${ }^{2}$ Department of Epidemiology, Brown University School of Public Health, Providence, RI, USA. ${ }^{3}$ AMPATH Partnership, Eldoret, Kenya. ${ }^{4}$ Division of Medicine, Moi Teaching and Referral Hospital, Eldoret, Kenya. ${ }^{5}$ Department of Medicine, Vanderbilt University, Nashville, TN, USA. ${ }^{6}$ Department of Statistics, North Dakota State University, Fargo, ND, USA. 'Department of Medicine, Indiana University, Indianapolis, USA. ${ }^{8}$ Department of Medicine, Duke Clinical Research Institute and Duke Global Health Institute, Duke University, Durham, NC, USA.

\section{Received: 12 June 2015 Accepted: 29 September 2015} Published online: 14 October 2015

\section{References}

1. Joint United Nations Programme on HIV/AIDS (UNAIDS). UNAIDS Report on the Global AIDS Epidemic 2013. Geneva: UNAIDS; 2013.

2. Mensah GA, Roth GA, Sampson UK, Moran AE, Feigin VL, Forouzanfar MH, et al. Mortality and Causes of Death collaborators. Mortality from cardiovascular diseases in sub-Saharan Africa, 1990-2013: a systematic analysis of data from the Global Burden of Disease Study 2013. Cardiovasc J Afr. 2015;1:S6-S10.3.

3. Mensah GA, Moran AE, Roth GA, Narula J. The global burden of cardiovascular diseases,1990-2010. Glob Heart. 2014;9:183-4.

4. Triant VA, Lee H, Hadigan C, Grinspoon SK. Increased acute myocardial infarction rates and cardiovascular risk factors among patients with human immunodeficiency virus disease. J Clin Endocrinol Metab. 2007;92:2506-12.

5. Lang S, Mary-Krause M, Cotte L, et al. French Hospital Database on HIV-ANRS CO4. Increased risk of myocardial infarction in HIV-infected patients in France, relative to the general population. AIDS. 2010;24:1228-30.

6. Law MG, Friis-Møller N, El-Sadr WM, et al. D:A:D Study Group. The use of the Framingham equation to predict myocardial infarctions in HIV-infected patients: comparison with observed events in the D:A:D Study. HIV Med. 2006;7:218-30.

7. Strategies for Management of Antiretroviral Therapy (SMART) Study Group, El-Sadr WM, Lundgren J, Neaton JD. CD4+count-guided interruption of antiretroviral treatment. N Engl J Med. 2006;355(22):2283-96.

8. Paisible AL, Chang CC, So-Armah KA, et al. HIV infection, cardiovascular disease risk factor profile, and risk for acute myocardial infarction. J Acquir Immune Defic Syndr. 2015;68:209-16.

9. Saves M, Chene G, Ducimetiere P. Risk factors for coronary heart disease in patients treated for human immunodeficiency virus infection compared with the general population. Clin Infect Dis. 2003;37:292-8.

10. Freiberg MS, Chang CC, Kuller LH, et al. HIV infection and the risk of acute myocardial infarction. JAMA Int Med. 2013;173:614-22.

11. Bloomfield GS, Hogan JW, Keter A, et al. Hypertension and obesity as cardiovascular risk factors among HIV seropositive patients in Western Kenya. PLoS One. 2011;6, e22288.

12. Peck RN, Shedafa R, Kalluvya $S$, et al. Hypertension, kidney disease, HIV and antiretroviral therapy among Tanzanian adults: a cross-sectional study. BMC Med. 2014;12:125.

13. Zhou DT, Kodogo V, Chokuona KF, et al. Dyslipidemia and cardiovascular disease risk profiles of patients attending an HIV treatment clinic in Harare, Zimbabwe. HIV AIDS (Auckl). 2015;7:145-55.

14. Schoffelen AF, de Groot E, Tempelman HA et al. Carotid intima media thickness in HIV-infected, mainly female subjects in rural South Africa is related to traditional cardiovascular risk factors but not to HIV-related factors. Clin Infect Dis. 2015 Jul 27. Ahead of print.

15. Wilcox S, Stefanick ML. Knowledge and perceived risk of major diseases in middle-aged and older women. Health Psychol. 1999;18:346-53.

16. Becker MH, Maiman LA, Kirscht JP, Haefner DP, Drachman RH. The Health Belief Model and prediction of dietary compliance: a field experiment. J Health Soc Behav. 1977;18:348-66.

17. McKinley S, Dracup K, Moser DK, et al. International comparison of factors associated with delay in presentation for AMI treatment. Eur J Cardiovasc Nurs. 2004;3:225-30.

18. Choi S, Rankin S, Stewart A, Oka R. Perceptions of coronary heart disease risk in Korean immigrants with type 2 diabetes. Diabetes Educ. 2008;34:484-92.
19. BeLue R, Okoror TA, Iwelunmor J, et al. An overview of cardiovascular risk factor burden in sub-Saharan African countries: a socio-cultural perspective. Global Health. 2009;5:10.

20. NASCOP Kenya AIDS indicator survey 2012. http://nascop.or.ke (October 2, 2015).

21. Inui TS, Nyandiko WM, Kimaiyo SN, et al. AMPATH: living proof that no one has to die from HIV. J Gen Intern Med. 2007;22:1745-50.

22. Einterz RM, Kimaiyo S, Mengech HNK, et al. Responding to the HIV pandemic: the power of an academic medical partnership. Acad Med. 2007:82:812-8.

23. Nabel EG, Stevens $S$, Smith R. Combating chronic disease in developing countries. Lancet. 2009;373:2004-6.

24. Bloomfield GS, Kimaiyo S, Carter EJ, et al. Chronic noncommunicable cardiovascular and pulmonary disease in sub-Saharan Africa: An academic model for countering the epidemic. Am Heart J. 2011;161:842-7.

25. NASCOP Kenya guidelines for antiretroviral drug therapy, $4^{\text {th }}$ edition 2011. http://healthservices.uonbi.ac.ke/sites/default/files/centraladmin/ healthservices/Kenya\%20Treatment\%20Guidelines\%202011.pdf (Accessed August 15,2015).

26. Vaidya A, Aryal UR, Krettek A. Cardiovascular health knowledge, attitude and practice/behaviour in an urbanising community of Nepal: a population-based cross-sectional study from Jhaukhel-Duwakot Health Demographic Surveillance Site. BMJ Open. 2013;3, e002976.

27. Mosca L, Jones WK, King KB, Ouyang P, Redberg RF, Hill MN. Awareness, perception, and knowledge of heart disease risk and prevention among women in the United States. American Heart Association Women's Heart Disease and Stroke Campaign Task Force. Arch Fam Med. 2000;9:506-15.

28. Gill R, Chow CM. Knowledge of heart disease and stroke among cardiology inpatients and outpatients in a Canadian inner-city urban hospital. Can J Cardiol. 2010;26:537-40.

29. WHO STEPS Instrument (Core and Expanded): The WHOSTEPwise approach to chronic disease risk factor surveillance (STEPS). http://www.who.int/chp/ steps/STEPS_Instrument_v2.1.pdf. (Accessed August 15, 2015)

30. American Heart Association. http://www.americanheart.org/ (Accessed February 11, 2015).

31. Smith Jr SC, Benjamin EJ, Bonow RO, et al. World Heart Federation and the Preventive Cardiovascular Nurses Association. AHA/ACCF Secondary Prevention and Risk Reduction Therapy for Patients with Coronary and other Atherosclerotic Vascular Disease: 2011 update: a guideline from the American Heart Association and American College of Cardiology Foundation. Circulation. 2011;124:2458-73.

32. Friis-Moller N, Reiss $P$, Sabin CA, et al. Class of antiretroviral drugs and the risk of myocardial infearctio. N Engl J Med. 2007;356:1723-35.

33. Kamitani E, Fukuoka Y, Dawson-Rose C. Knowledge, Self-efficacy, and Self-perceived Risk for Cardiovascular Disease among Asians Living With HIV: The Influence of HIV Stigma and Acculturation. J Assoc Nurses AIDS Care. 2015;26(4):443-53.

34. Jafary FH, Aslam F, Mahmud H, et al. Cardiovascular health knowledge and behavior in patient attendants at four tertiary care hospitals in Pakistan-a cause for concern. BMC Public Health. 2005;5:124.

35. Mukattash TL, Shara M, Jarab AS, Al-Azzam SI, Almaaytah A, Al Hamarneh YN. Public knowledge and awareness of cardiovascular disease and its risk factors: a cross-sectional study of 1000 Jordanians. Int J Pharm Pract. 2012;20:367-76.

36. Cioe PA, Crawford SL, Stein MD. Cardiovascular risk-factor knowledge and risk perception among HIV-infected adults. J Assoc Nurses AIDS Care. 2014;25:60-9.

37. Bloomfield GS, Baldridge A, Agarwal A, et al. Disparities in cardiovascular research output and citations from 52 african countries: a time-trend, bibliometric analysis (1999-2008). J Am Heart Assoc. 2015;4.

38. Awad A, Al-Nafisi H. Public knowledge of cardiovascular disease and its risk factors in Kuwait: a cross-sectional survey. BMC Public Health. 2014;14:1131.

39. Saeed O, Gupta V, Dhawan N, et al. Knowledge of modifiable risk factors of Coronary Atherosclerotic Heart Disease (CASHD) among a sample in India. BMC Int Health Hum Rights. 2009;9:2. 